\title{
Qué hace un docente de Ingeniería para promover la participación de sus alumnos en la construcción de conocimientos: escritura, revisión colectiva y diálogo sobre lo escrito ${ }^{1}$
}

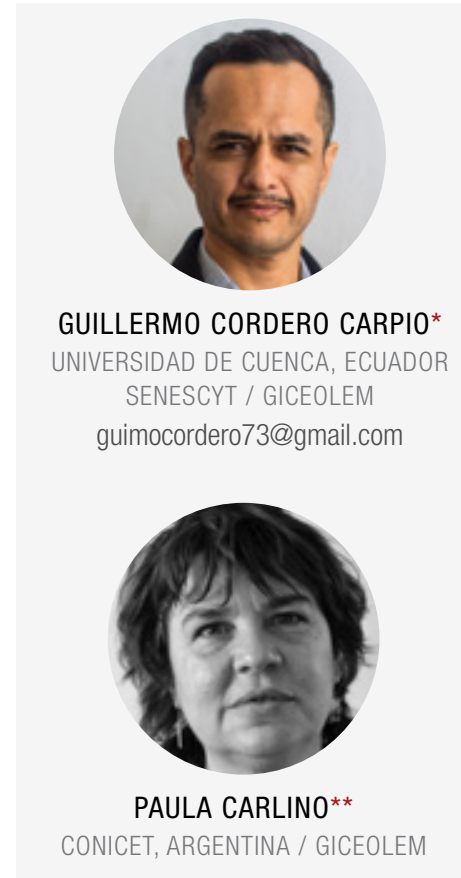

\author{
What does an engineering teacher do to \\ promote the participation of his students in the \\ construction of knowledge: writing, collective \\ revision and dialogue about writing
}

Recibido: 24 de mayo de 2019 | Aprobado: 10 de junio de 2019

\section{Resumen}

Este artículo forma parte de una tesis doctoral en curso que estudia el proceso que atraviesa un docente cuando procura incluir en sus clases la escritura como herramienta para enseñar los contenidos de la asignatura de Métodos numéricos. La investigación, de carácter intervencionista, se realizó a partir de dos ejes complementarios. Un eje formativo en el cual el docente trabajó junto a un investigador-colaborador en el diseño, implementación y rediseño de una secuencia didáctica centrada en la escritura de una entrada de manual. Y un eje investigativo, que tuvo como propósito describir los cambios que se produjeron en la actividad del docente y de los alumnos en el aula, y las conceptualizaciones del docente que acompañaron dichos cambios, a lo largo de tres implementaciones de la secuencia. Se videograbaron 10 horas de clase y se audiograbaron 30 horas de sesiones de trabajo conjunto y entrevistas al profesor; en ambos casos los registros fueron transcritos. En este artículo, nos centramos en las acciones del profesor que abrieron oportunidades para

\footnotetext{
1 Este trabajo se deriva de la tesis doctoral en curso del primer autor, dirigida por la segunda autora, e integra dos proyectos de Investigación: PICTO 2016-06: La actividad docente situada. Usos de la lectura y la escritura como herramientas mediadoras de aprendizajes en la enseñanza de las disciplinas en nivel secundario y superior (cofinanciado por la Universidad Pedagógica Nacional y la Agencia Nacional de Promoción Científica y Técnica -ANPCyT- de Argentina), y Proyecto PICT-2014-2793 financiado por la ANPCyT: Prácticas de enseñanza que promueven u obstaculizan la participación y el aprendizaje a través de la lectura y la escritura en diferentes materias de la educación secundaria y superior, ambos dirigidos por Paula Carlino. Los análisis presentados han sido discutidos en las reuniones del GICEOLEM. Otra publicación de la misma tesis, con resultados que complementan los aquí expuestos, puede encontrarse en: Cordero Carpio, G. y Carlino, P. (2019). El análisis de la actividad docente: un medio para reflexionar sobre el potencial epistémico de la escritura en una materia de Ingeniería. En C. Bazerman, D. Russell, P. Rogers, B. González, L. Peña, E. Narváez, P. Carlino, M. Castelló y M. Tapia (Comps.) Conocer la escritura: investigación más allá de las fronteras. Bogotá: Pontificia Universidad Javeriana. Próximamente disponible en https://sites.google.com/ site/giceolem2010/universidad
}

\footnotetext{
* Magister en Lingüística y Lexicografía Hispánica, Real Academia de la Lengua Española - Universidad de León, España; Magister en Estudios de la Cultura Mención Literatura Hispanoamericana, Universidad Andina Simón Bolívar sede Quito, Ecuador; y Licenciado en Ciencias de la Educación con Especialización en Lengua y Literatura Española, Universidad de Cuenca, Ecuador. Actualmente, se desempeña como docente-investigador en la Universidad de Cuenca, Ecuador, y cursa el programa de Doctorado en Ciencias de la Educación de la Universidad Nacional de La Plata, Argentina. Para contactar al autor: guillermo.cordero@ucuenca.edu.ec

** Doctora en Psicología por la Universidad Autónoma de Madrid y Licenciada en Psicología por la Universidad de Buenos Aires. Se desempeña como Investigadora Principal del CONICET en el Instituto de Lingüística de la Universidad de Buenos Aires y como Profesora de la Universidad Pedagógica Nacional, de Argentina. Dirige el GICEOLEM (https://sites.google.com/site/giceolem2010/), equipo pluridisciplinar que investiga las relaciones entre enseñar, aprender, leer y escribir en diversas disciplinas del nivel secundario, superior y de posgrado. Muchas de sus publicaciones pueden accederse libremente desde https://www.aacademica.org/paula.carlino/
} 
que los estudiantes participasen en el diálogo sobre lo escrito, ejercieran quehaceres de revisión y, de esta manera, aportasen a la construcción de conocimientos en el aula. Los resultados muestran que hubo cambios de una implementación a otra. El profesor ensayó diferentes estrategias para abrir y regular los ciclos de revisión, que dieron paso a una mayor y más variada participación de los alumnos en la construcción conjunta de conocimientos. La caracterización de estas estrategias es el foco del presente artículo.

Palabras clave: escribir a través del currículo, formación docente, diálogo sobre lo escrito, ingeniería

\begin{abstract}
This article is part of an ongoing doctoral thesis that studies the process that a teacher goes through when trying to include writing as a tool to teach the contents of the subject of Numerical methods. The study is carried out from two complementary axes. In the formative axis, the teacher worked together with the researcher-collaborator in the design and implementation of a teaching sequence centered on the writing of a user manual. In the research axis, the purpose was to describe the changes that took place in the teaching activity and the teacher's conceptualizations about these changes, along three implementations of the sequence in successive semesters. 10 hours of class were video-recorded and 30 hours of interviews were audio-recorded, and transcribed in both cases. In this paper, we analyze the teacher's actions, which opened opportunities for students to participate in the dialogue about writing, to perform tasks of revision and to contribute to the construction of knowledge in the classroom. The results show changes from one implementation to another. The professor tried different strategies to open and regulate the revision cycles that gave way to a greater and more varied participation of the students in the joint construction of knowledge. The characterization of these strategies is the focus of this article.
\end{abstract}

Keywords: writing across the curriculum, teacher education, talking about text, engineering

\section{Introducción}

El proyecto de investigación doctoral que da origen a este artículo nació a partir del encuentro de los autores con Francisco, profesor de ingeniería de una destacada universidad pública de Ecuador, en el marco de un congreso sobre lectura y escritura en las disciplinas. Francisco estaba preocupado por los bajos resultados obtenidos a partir de una tarea centrada en la escritura de una entrada de manual de usuario, con la cual venía trabajando desde hacía algún tiempo en sus clases de Métodos numéricos. Por ello, manifestó su deseo y disponibilidad para iniciar un proceso colaborativo con el fin de abordar este problema. Por su lado, los autores, basados en los aportes de la corriente Escribir a través del curriculum (Bazerman et. al., 2005), la enseñanza mediante el diálogo (Dysthe, Bernhardt y Esbjørn, 2013), la didáctica de las prácticas del lenguaje (Lerner, 2001), la teoría de las situaciones didácticas (Brousseau, 2007; Sensevy, 2007) y la didáctica profesional (Pastré, 2007; 2008; 2011), deseaban explorar la actividad docente orientada a favorecer el uso de la escritura como herramienta epistémica.

De este modo, colaborativamente diseñaron una secuencia didáctica para guiar la escritura de la entrada de manual y aprovechar su potencial epistémico (su uso como herramienta de aprendizaje de
Ios contenidos disciplinares); se tuvo en cuenta que una de las condiciones para que escribir contribuya a la elaboración del conocimiento es propiciar el diálogo reflexivo sobre lo escrito. La secuencia fue implementada, observada y videograbada en tres ocasiones, a lo largo de tres semestres. Después de cada implementación, se realizaron entrevistas de autoconfrontación que, a través de la visualización de fragmentos de video, permitieron que el profesor pudiera reflexionar sobre lo ocurrido en la implementación anterior a fin de realizar ajustes para la del siguiente semestre.

Los resultados que se exponen en este artículo responden al objetivo de caracterizar, a partir de transcripciones de clase, las estrategias que ensayó el docente a lo largo de las tres implementaciones para promover la participación de los alumnos en el diálogo sobre lo escrito durante la revisión colectiva de borradores (textos provisorios) de la entrada de manual. En el apartado 1 presentamos la metodología, que incluye la descripción del trabajo de codiseño didáctico, y en el 2, los resultados del análisis de la actividad del docente y los estudiantes en el aula. El 3 lo reservamos para las conclusiones.

\section{Metodología}

La presente investigación se planteó como un estudio de caso de carácter intervencionista, de codiseño 
didáctico, confrontativo y longitudinal. Intervencionista, en tanto coadyuvó a provocar un fenómeno inusual en clase para poder estudiarlo. De codiseño, porque la secuencia didáctica fue planificada conjuntamente entre el profesor y los investigadores, durante numerosas reuniones entre el docente y el primer autor, y entre el primer autor (tesista) y la segunda autora (directora de tesis). Confrontativo, porque después de cada implementación se mantuvieron entrevistas de autoconfrontación en las que el docente tuvo ocasión de reflexionar sobre su actividad a partir del análisis de fragmentos de videograbación de clases seleccionados por el primer autor. Longitudinal, dado que las clases y entrevistas fueron iteradas en tres semestres consecutivos para examinar cambios a lo largo de este tiempo. Las clases fueron videograbadas, en tanto las sesiones de trabajo de codiseño y las entrevistas de autoconfrontación fueron audiograbadas; en ambos casos los registros fueron posteriormente transcritos y completados con las notas del investigador. Para el análisis de datos se empleó una estrategia mixta: cualitativa y cuantitativa.

\section{Contexto de partida}

El profesor, un ingeniero con doctorado y posdoctorado fuera de su país, impartía la asignatura de Métodos numéricos en el tercer semestre de la carrera de Ingeniería Civil, con una carga horaria de 4 horas semanales (64 semestrales), y un promedio de 25 estudiantes. Según el programa, la asignatura perseguía un objetivo práctico: capacitar a los alumnos como implementadores y usuarios de métodos numéricos. El profesor había incluido la escritura de entradas de manual como una de las actividades orientadas a la consecución de este objetivo. Consideraba que escribir una entrada de manual abría oportunidades para enseñar a los estudiantes a discriminar y manejar la información que se necesita para usar un método numérico.
Sin embargo, un primer análisis realizado de manera conjunta con el profesor, en una sesión de trabajo preparatoria del codiseño, anterior a la primera implementación, mostró que la forma en la cual venía realizando esta tarea en sus clases no era consistente con el objetivo que se proponía lograr a través de ella. Al terminar la clase magistral de cada método, el profesor planteaba a sus estudiantes la escritura de la respectiva entrada de manual, como una tarea domiciliaria (Fig 1, rectángulos blancos). Para orientarla, se limitaba a intervenir "en los extremos" (Carlino, 2004; Carlino, Iglesia y Laxalt, 2013): al principio, con una consigna general y, al final, con la corrección y calificación de la primera producción recibida. Según él mismo manifestó en ese momento:

Para serte sincero lo que he hecho es decirles 'quiero estos ítems [enumera algunas secciones del género entrada de manual]: el Propósito, la Sintaxis, la Descripción...' La verdad, actúo de forma bien brutal.

De esta manera, al dejar el proceso de escritura por fuera de la clase y a cargo de los estudiantes, dando por descontado que podrían realizarlo solos, el profesor proponía el uso de la escritura como si se tratara solo de un canal para registrar y comunicar nociones ya pensadas por ellos a priori. De este modo, se desaprovechaba la potencialidad del proceso de escritura (que involucra generar ideas, textualizarlas y revisarlas) de servir, en ciertas condiciones, como un medio de construcción de conocimientos. A este fin se encaminó nuestro trabajo colaborativo.

\section{Codiseño de una secuencia didáctica que potencie la función epistémica de la escritura}

Los registros de la sesión de trabajo preparatoria, referida en el apartado anterior, muestran que las condiciones en la cuales el docente gestionaba

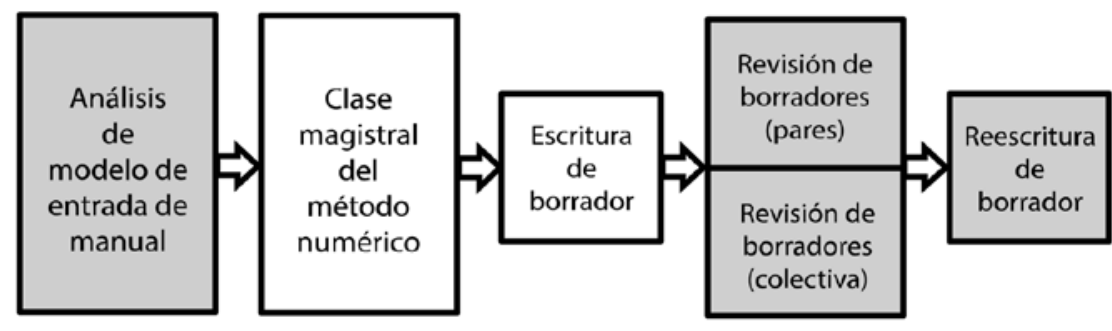

Figura 7. Secuencia didáctica para orientar la escritura de la entrada de manual. 
la tarea de escritura distaban de las señaladas por la investigación como necesarias para que la escritura se constituya en instrumento de aprendizaje, especialmente porque carecían de diálogo plural (Dysthe, 1996; Dysthe et. al., 2013), de controversia académica, de colaboración, en fin, de intercambios para reconsiderar ideas (Carter, Ferzli y Wiebe, 2007; Klein, 1999; Langer y Applebee, 2007; Ochsner y Fowler, 2004). De igual modo, nos percatamos de que la modalidad de enseñanza exclusivamente expositiva se alejaba de lo señalado por la investigación didáctica, que muestra que, para aprender, los alumnos necesitan involucrarse en la construcción de conocimientos y no ser meros receptores de saberes ajenos. En este sentido, nos interesó discutir con el docente la conveniencia, según lo expresa Lerner (2017), de compartir con ellos la responsabilidad del aprendizaje, superar la ficción de que el tiempo del aprendizaje coincide con el tiempo de la enseñanza, articular diferentes formas de organización de la clase y generar un ambiente de trabajo en el que los alumnos se sintieran autorizados a expresar lo que genuinamente piensan acerca del objeto de conocimiento.

Para acercar el quehacer del docente a estas condiciones, codiseñamos una secuencia didáctica orientada a intensificar, dentro del aula, la labor conjunta entre el profesor y los alumnos en torno al proceso de escritura de la entrada de manual. Procuramos enfatizar el "aspecto constructivo" (registro epistémico) de la actividad en clase, que habitualmente queda relegado por el "aspecto productivo" (registro pragmático) (Pastré, 2008). Efectivamente, cuando lo que prevalece es la enseñanza expositiva a cargo del docente, y el avance rápido de los contenidos a cubrir en el programa, lo que se resiente es lo que van comprendiendo los alumnos sobre esa sucesión de temas, así como lo que va comprendiendo el docente sobre lo que comprenden sus alumnos.

El trabajo de codiseño didáctico se inspiró en la ingeniería didáctica (Artigue, 1995) y la ingeniería cooperativa (Sensevy, Forest, Quilio y Morales, 2013), aunque sin ajustarse totalmente a estos enfoques. De ellos, tomó el proceso iterativo a través del cual un colectivo de profesores e investigadores diseñan, implementan y re-implementan (después de haber analizado y evaluado la práctica previa) una unidad de enseñanza de un tema en particular $^{2}$. Tanto el docente como los investigadores compartieron el rol de construir dialógicamente la secuencia didáctica: el docente asumió la potestad de definirla, haciéndose cargo de las decisiones finales tanto en el diseño como en la implementación de la secuencia; mientras que el primer autor asumió el rol de colaborador, poniendo sobre la mesa recursos didácticos y herramientas interpretativas que pudieran resultarle útiles.

Como parte del codiseño, se agregaron a la secuencia habitual (Fig. 1, rectángulos blancos) dos clases y una tarea. En primer lugar, se incluyó una clase previa de dos horas dedicada al análisis de un modelo de entrada de manual (izquierda, en gris). Con esta actividad se pretendía que los estudiantes tuvieran una representación de las características del género "entrada de manual", su estructura, el tipo de información que se presenta en cada sección, los distintos tipos de lenguaje que utiliza, el destinatario al cual va dirigido (usuario del método numérico) y su propósito (ofrecer instrucciones de uso). Asimismo, en esta actividad el docente debía establecer, de manera consensuada con los estudiantes, los criterios de producción y evaluación del escrito.

En segundo lugar, se incluyó una clase posterior para la revisión de borradores de la entrada de manual y una segunda tarea domiciliaria (derecha, rectángulos grises). Estas actividades tuvieron como propósito evitar que la primera producción de los estudiantes fuera solo un documento para acreditar su conocimiento y, en cambio, resultara un texto intermediario desde el cual discutir y repensar los contenidos de Métodos numéricos. De esta manera, la revisión -habitualmente llevada a cabo como una operación de control, realizada exclusivamente por el profesor una vez que los estudiantes habían finalizado el proceso de escritura- pasó a ser concebida como una actividad de enseñanza. En ella, el profesor se propuso dos objetivos: por un lado, acompañar a los estudiantes para que participaran en la práctica de revisión (que forma parte integral del proceso de escritura); por otro lado, y gracias a esta participación, propiciar que pudieran desarrollar (ampliar, confrontar, cuestionar, ajustar) su conocimiento sobre el método numérico objeto de estudio.

\footnotetext{
2 Otros aspectos de la ingeniería didáctica, como la validación interna basada en la confrontación entre el análisis a priori y el análisis a posteriori, fueron dejados de lado en el análisis y, en cambio, se apeló al dispositivo de la autoconfrontación del profesor con las huellas de su actividad propio de la didáctica profesional.
} 
A su vez, dentro de la clase de revisión de borradores, se consideraron dos actividades que entramaran escritura y oralidad: una revisión entre pares, en la cual cada grupo de estudiantes debía discutir y comentar el borrador de otro grupo ${ }^{3}$; y una posterior revisión colectiva, en la cual el docente y los estudiantes revisarían de manera conjunta los borradores de la entrada de manual. Según Wells (1990, 2004), las prácticas en torno a la escritura de textos involucran actividades mentales complejas que necesitan aprenderse por medio de la participación en eventos de lectura y escritura en los que estas actividades, habitualmente internas, se exterioricen a partir del diálogo entre el profesor y los alumnos (y entre alumnos), volviéndose disponibles para su apropiación.

Con el fin de asistir la práctica del profesor y de recoger datos que permitieran estudiar, desde una perspectiva longitudinal, los cambios en su actividad y las conceptualizaciones que los acompañan, la secuencia fue iterada en tres semestres consecutivos, observada y videograbada.

\section{Entrevistas de autoconfrontación}

Después de cada una de las implementaciones, se realizaron entrevistas de autoconfrontación (Fernández y Clot, 2010; Pastré, 2008, 2011). Estas tuvieron como finalidad ayudar al profesor a reflexionar, a través de la visualización de fragmentos de video, sobre lo ocurrido en la implementación anterior y a realizar ajustes para la implementación del siguiente semestre. En este sentido, la reflexión sobre la actividad docente se realizó desde una doble mirada: retrospectiva en tanto permitió hacer un balance, comprender lo que había funcionado o no, y prospectiva en tanto ayudó a anticipar y preparar la próxima implementación. Estas entrevistas funcionaron, a su vez, como un dispositivo dialógico de interacción triádica, que permitió al docente conceptualizar su actividad al interior de un espacio inter-subjetivo donde su versión pudo ser confrontada con las huellas de su actividad (el registro proveniente de la videofilmación) en el marco de una discusión con el investigador. Las sesiones de trabajo conjunto mediante entrevistas de autoconfrontación fueron audiograbadas y luego transcritas a los fines de un análisis que será objeto de otro artículo.

\section{Transformaciones en la actividad docente orientadas a promover la participación de los estudiantes en la revisión colectiva de borradores}

En este apartado, caracterizamos la actividad del profesor y cómo esta se transformó para gestionar la revisión colectiva de borradores que, a diferencia de sus clases habitualmente magistrales, precisaba de una práctica menos expositiva y más participativa, que promoviera la reflexión y el diálogo sobre lo escrito.

El análisis de las transcripciones de clase de las tres implementaciones nos permitió identificar que el profesor realizó dos movimientos complementarios orientados a transformar su modalidad de enseñanza predominantemente expositiva, registrada en la primera implementación, por una modalidad más participativa. Un movimiento de reticencia, con el cual se abstuvo de ejercer él mismo las operaciones de revisión, y otro movimiento de devolución y regulación, consistente en devolver a los alumnos el derecho y la responsabilidad de que sean ellos quienes ejerzan la revisión, y en regular ese ejercicio a través de acciones orientadas a reducir la incertidumbre de cómo hacerlo apropiadamente (Brousseau, 2007; Sensevy, 2007).

Nuestro análisis se desarrolló en tres etapas: en la primera segmentamos los registros de la revisión colectiva en episodios e identificamos los que preveían la participación de los estudiantes. En la segunda, segmentamos los episodios de revisión en ciclos, los cuantificamos e identificamos posibles cambios de una a otra implementación. Finalmente, en la tercera, caracterizamos las estrategias introducidas por el profesor que pudieron incidir en los cambios identificados.

\subsection{Estructura de participación de la revisión colectiva de borradores}

En la primera etapa del análisis, buscamos identificar la estructura de participación ${ }^{4}$ que siguió

\footnotetext{
3 La tarea de escritura de la entrada de manual fue realizada en pequeños grupos de tres o cuatro estudiantes, al igual que la revisión por pares. Por este motivo, cuando hablamos de autores o revisores pares, en realidad estamos hablando de grupos autores o grupos revisores pares.

4 Para analizar la interacción profesor-alumnos-texto, segmentamos las transcripciones de clase en unidades más pequeñas y manejables que nos permitieran identificar cómo se organizó la actividad. De esta forma, segmentamos la revisión colectiva en episodios, los episodios en ciclos y estos en acciones. En cada uno de estos segmentos tratamos de identificar la forma en que se estructuró la participación, es decir, de identificar el patrón de interacción en dicho segmento (Sánchez, García y Rosales, 2010).
} 
la actividad de revisión colectiva de borradores y, dentro de esta, los episodios que contemplaron la participación de los estudiantes. Para alcanzar este objetivo, segmentamos las transcripciones de la revisión colectiva en episodios, es decir, en fragmentos de actividad con finalidades y reglas de participación reconocibles y diferenciables (Sánchez, García y Rosales, 2010). En la Tabla 1, presentamos los resultados de esta primera operación.

Tabla 7. Episodios de la revisión colectiva de borradores, finalidad y estructura de interacción

\begin{tabular}{|l|c|c|}
\hline \multicolumn{1}{|c|}{ EPISODIOS } & FINALIDAD & ESTRUCTURA DE INTERACCIÓN \\
\cline { 1 - 1 } 1. Planificación & Definir la tarea & Monológica \\
\cline { 1 - 1 } 2. Título & & \\
\cline { 1 - 1 } 3. Propósito & & \\
\cline { 1 - 1 } 4. Sintaxis & Revisar borrador & Monológica/dialógica \\
\cline { 1 - 1 } 5. Descripción & \begin{tabular}{r}
$\mid$ \\
\cline { 1 - 1 } 6. Comentarios
\end{tabular} & \\
\cline { 1 - 1 } 7. Ejemplos & Concluir la tarea & Monológica \\
\cline { 1 - 1 } 8. Conclusión & & \\
\hline
\end{tabular}

Los resultados de la segmentación dejan ver que la revisión colectiva de borradores presentó una estructura de ocho episodios en las tres implementaciones, con una sola excepción: en la tercera implementación el episodio 7, Ejemplos, no llegó a realizarse por falta de tiempo. La estructura contempló un episodio de planificación (en el cual el profesor dio la consigna de la actividad), seis episodios de revisión de cada una de las secciones del género entrada de manual (Título, Propósito, Sintaxis, Descripción, Comentarios y Ejemplos), y un episodio de conclusión o cierre de la actividad realizada (que incluyó la consigna de la reescritura del borrador como tarea domiciliaria). Los episodios identificados se distinguen de acuerdo con su finalidad y con la estructura de participación que siguieron. Por un lado, están los episodios centrales que tuvieron como función revisar colectivamente cada una de las secciones de la entrada de manual. Si bien, en el codiseño, la revisión de borradores fue planificada como una actividad conjunta entre el profesor y los alumnos, no todos los episodios de revisión contaron con la participación de los estudiantes, es decir, no todos tuvieron una estructura de interacción dialógica. Por otro lado, están los episodios marco que tuvieron por finalidad definir la tarea (episodio de planificación) o finalizarla (episodio de conclusión), estos episodios tuvieron siempre una estructura de participación monológica, es decir, estuvieron exclusivamente a cargo del profesor y no consideraron la participación de los alumnos.

De aquí en adelante, nos centraremos solamente en los episodios de revisión que preveían la participación de los estudiantes (se diera o no esta participación en cada una de las implementaciones).

\subsection{Hacia una modalidad más participativa}

Para identificar los cambios que se dieron entre una y otra implementación relacionados con la interacción profesor-alumno-borradores, segmentamos los registros de los episodios de revisión en ciclos, es decir, en conjuntos de acciones (o actos de habla) centrados en la revisión de un aspecto (formal y/o de contenido) de las diferentes secciones de la entrada de manual. Una vez segmentados los ciclos, los clasificamos según fueran monológicos (manejados exclusivamente por el profesor ${ }^{5}$ ) o dialógicos (trabajados conjuntamente por el profesor y los alumnos ${ }^{6}$ ). Finalmente, cuantificamos cuántos ciclos monológicos y cuántos dialógicos se realizaron en cada implementación.

Los resultados, que se presentan en la Tabla 2, muestran una tendencia creciente en los ciclos dialógicos (Dia.) que pasan del $28.5 \%$ en la primera implementación, al $43.7 \%$ en la segunda y al $71.8 \%$ en la tercera; frente a una tendencia decreciente inversamente proporcional en los ciclos monológicos (Mon.).

Estos resultados muestran que en la primera implementación la actividad de revisión siguió, en

5 Llamamos ciclos monológicos a aquellos en los que el profesor es el único que interviene oralmente para revisar el borrador. No obstante, es necesario tener en cuenta que la participación de los alumnos, más allá de la interacción oral, está siempre presente a través de los borradores previamente revisados por los grupos de pares. En este sentido, todos los ciclos de revisión cuentan con la "participación escrita" de los estudiantes.

6 Cuando hablamos de ciclos dialógicos nos referimos a ciclos en los cuales dos o más personas se comunican oralmente. Para el efecto nos remitimos a la noción de "dialogismo" propuesta por Bajtin (2012) y a uno de sus desarrollos posteriores en el campo pedagógico, la noción de "aula con pluralidad de voces" propuesta por Olga Dysthe $(1996,2013)$. 
Tabla 2. Ciclos monológicos y dialógicos por episodio e implementación

\begin{tabular}{|l|r|r|r|r|r|r|r|r|r|}
\hline & \multicolumn{3}{|c|}{ IMPLEMENTACIÓN 1 } & \multicolumn{3}{|c|}{ IMPLEMENTACIÓN 2 } & \multicolumn{2}{|c|}{ IMPLEMENTACIÓN 3 } \\
\hline & Mon. & Dia. & TOTAL & Mon. & Dia. & TOTAL & Mon. & Dia. & TOTAL \\
\hline Título & 2 & 0 & 2 & 1 & 1 & 2 & 1 & 3 & 4 \\
\hline Propósito & 2 & 0 & 2 & 0 & 6 & 6 & 0 & 6 & 6 \\
\hline Sintaxis & 3 & 0 & 3 & 4 & 0 & 4 & 1 & 4 & 5 \\
\hline Descripción & 8 & 7 & 15 & 11 & 3 & 14 & 5 & 1 & 6 \\
\hline Comentarios & 3 & 0 & 3 & 2 & 0 & 2 & 2 & 9 & 11 \\
\hline Ejemplos & 2 & 1 & 3 & 0 & 4 & 4 & - & - & - \\
\hline Total & 20 & 8 & 28 & 18 & 14 & 32 & 9 & 23 & 32 \\
\hline$\%$ & $71.5 \%$ & $28.5 \%$ & $100 \%$ & $56.3 \%$ & $43.7 \%$ & $100 \%$ & $28.2 \%$ & $71.8 \%$ & $100 \%$ \\
\hline
\end{tabular}

gran medida, la modalidad habitual con la cual el profesor acostumbraba a impartir la asignatura: la clase expositiva. A pesar de que en el codiseño de la secuencia se había acordado que la actividad de revisión colectiva se realizaría como una situación de "enseñanza a través de un medio", que promoviera en el estudiante la construcción de conocimientos (Brosseau, 2007), esta se realizó, en su mayor parte, a partir de una situación de "enseñanza por lección", es decir, por transmisión del saber a cargo del profesor (Pastré, 2008). De acuerdo con su modelo operativo habitual, en la primera implementación el profesor llevó a cabo -como experto en los contenidos y en el discurso de la disciplina- la revisión colectiva de borradores, dejando poco espacio para que esta pudiera ser ejercida por los alumnos durante el plenario.

No obstante, el incremento de los ciclos dialógicos a partir de la segunda implementación muestra que el profesor se animó a realizar movimientos orientados a compartir con los alumnos el rol de revisores de textos, que habitualmente se había reservado para sí. Por un lado, asumió una actitud de mayor reticencia, es decir, fue capaz de "esconder parte de lo que sabía", para que fueran los alumnos quienes construyeran conocimiento en dirección de ese saber erudito (Sensevy, 2007). Por otro lado, ensayó diferentes estrategias para promover la participación de los estudiantes en la revisión de borradores, distanciándose progresivamente de la modalidad expositiva registrada en la primera implementación.
A continuación, mediante el análisis de cuatro ciclos de revisión, enfocamos las estrategias que ensayó el docente para llevar a cabo la revisión colectiva de borradores de la entrada de manual.

\subsection{Estrategia expositiva y estrategias participativas}

En la tercera etapa de análisis, caracterizamos las estrategias que puso en práctica el docente para llevar adelante la revisión colectiva de entradas de manual. A los fines de este artículo, consignamos los resultados a partir del análisis de cuatro ciclos representativos: uno monológico y tres dialógicos. El análisis del ciclo monológico nos permitirá ilustrar la que llamamos estrategia expositiva; mientras que el análisis de los ciclos dialógicos ilustrará tres tipos de estrategias participativas empleadas por el profesor.

\subsubsection{Estrategia expositiva}

Los ciclos monológicos en las tres implementaciones se caracterizaron por un patrón de interacción diádico profesor-borradores, realizado a partir de la combinación de tres acciones: Lee borrador, Revisa (diagnostica y/o propone solución) y Confirma. En la Tabla 3, analizamos, como ejemplo ilustrativo, un ciclo en el que se combinan las tres acciones (hay ciclos que presentan dos o, incluso, una). Este ciclo forma parte del episodio dedicado a la revisión de la sección Sintaxis, en la cual los estudiantes debían consignar, en lenguaje de programación (código Matlab) ${ }^{7}$, la "llamada de la función", es decir, la

7 Una de las características de la entrada de manual es su multimodalidad, es decir, la utilización simultánea de cuatro sistemas semióticos: lenguaje verbal, de programación (código Matlab), matemático y gráfico. Para distinguir los tres primeros se utiliza diferente tipografía. 
Tabla 3. Ciclo monológico, sección Sintaxis, implementación 1

\begin{tabular}{|l|l|l|c|}
\hline & \multicolumn{1}{|c|}{ TRANSCRIPCIÓN ${ }^{9}$} & \multicolumn{1}{|c|}{ ACCIONES } & BORRADOR \\
\hline 1 & $\begin{array}{l}\text { Profesor: Ahora lo de la Sintaxis: [punto, iteraciones] [lee y lo transcribe del } \\
\text { borrador que tiene en sus manos a la pizarra], }\end{array}$ & Lee borrador & Confirma (contenido) \\
\hline 2 & P: bueno es básicamente lo que les pedí, ¿no es cierto? & [punto, iteraciones] \\
\hline 3 & $\begin{array}{l}\text { P: Tal vez ahí yo sí les pediría elegir nombres más intuitivos, "punto" está un poco } \\
\text { Como no muy intuitivo, "iteraciones" me parece que sí se entiende mejor. Y [elijan } \\
\text { nombres] más cortos, traten de... Fíjense en las entradas [de manual] de Matlab, } \\
\text { por lo general no tienen palabras completas. }\end{array}$ & Revisa (forma) & \\
\hline
\end{tabular}

fórmula mediante la cual el usuario le ordena al programa que ponga en marcha la resolución del problema que quiere resolver ${ }^{8}$.

El profesor abrió el ciclo con la lectura de dos datos de la sección Sintaxis (turno 1 en Tabla 3) y acto seguido realizó, él mismo, dos acciones que completaron y cerraron la revisión. En primer lugar, confirmó el contenido de la respuesta (2). Y, en segundo lugar, revisó la forma en la cual estaba expresada (3): diagnosticó un problema relacionado con el lenguaje de programación, el nombre del primer dato "está un poco como no muy intuitivo"; y, si bien no propuso una solución, explicó las características que esta debía tener: los nombres debían ser "más intuitivos" y "más cortos". Completó la acción remitiendo a los estudiantes al manual de Matlab, que citó como modelo del género.

En este ciclo, puede notarse que el profesor realizó él mismo la tarea de revisión sin invitar a los alumnos a participar de ella. Después de leer el borrador, no intentó ceder la palabra y ejerció los quehaceres de revisión por sí solo. Los alumnos actuaron como observadores. Si bien el profesor estableció un diálogo oculto ${ }^{10}$ que se verifica en una expresión mediante la cual corroboró que los alumnos seguían el hilo de su discurso: "¿no es cierto?" o en el apelativo "fijense", estos no fueron convocados a participar en la revisión colectiva del borrador.
Se trató, como se puede ver, de una situación de enseñanza transmisiva y, más específicamente, por demostración del procedimiento de revisión. En este caso, a falta de acciones de revisión de los estudiantes, la tarea cumplió un fin ilustrativo, sin llegar a constituirse en un medio que promoviera su participación. La estrategia expositiva del profesor hizo que la actividad se concentrara más en la revisión del texto escrito y menos en ayudar a desarrollar prácticas de revisión por parte de los alumnos. Puede observarse que, sin participación de los estudiantes, el profesor dispuso, en este y en el resto de ciclos monológicos, de un mayor control del tiempo didáctico que el que sostuvo en los ciclos dialógicos. De este modo, pudo completar la revisión de todas las secciones de la entrada de manual.

Finalmente, cabe notar que en unos pocos ciclos monológicos el profesor introdujo alguna acción para invitar a los alumnos a participar, pero sin obtener respuesta. Esto sucedió sobre todo en la segunda y tercera implementación, lo que respalda nuestra observación de que hubo un cambio progresivo en la actividad del profesor, aunque este cambio no siempre tuviera eco en la actividad de los alumnos.

\subsubsection{Estrategias participativas}

A diferencia de los ciclos monológicos, los dialógicos se caracterizaron por un patrón de interacción triádico profesor-alumnos-borradores. Estos ciclos

\footnotetext{
8 La Sintaxis comprende tres tipos de datos: la función (el método numérico), los datos de entrada (los que debe ingresar el usuario) y los datos de salida (los que quiere obtener).

9 En la transcripción de los registros de clase utilizamos las siguientes convenciones: cursiva cuando se lee directamente el texto revisado; "comillas" cuando se cita o se refiere a un elemento del texto revisado; negrita cuando se propone una solución alternativa; [...] cuando omitimos un fragmento de la grabación original; [comentarios] cuando reponemos el contexto, acciones, gestos, tiempo, etc. Además, en la columna izquierda, numeramos los turnos de habla para poder identificarlos en el análisis. En la columna derecha, transcribimos el texto que se está revisando según aparece escrito en el borrador de los alumnos objeto de revisión.

10 Bajtín (2012) propone la noción de "dialogismo oculto" para referirse a una conversación en la cual el discurso del primer interlocutor (en nuestro caso el profesor) está determinado por la presencia de un segundo locutor (el estudiante) que se mantiene en silencio.
} 
dejan ver una diversificación en las acciones realizadas por el profesor para promover la participación de los estudiantes de modo que fueran ellos quienes ejercieran las operaciones de revisión. A continuación, caracterizamos las tres estrategias observadas que apuntan a este fin.

\section{Invitar a revisar}

En el ciclo dialógico que analizamos en la Tabla 4, el profesor puso en práctica una estrategia simple, pero no por ello menos efectiva: invitar a los estudiantes a revisar colectivamente el borrador. Este ciclo forma parte del episodio dedicado a la revisión de la sección Descripción, en la cual los estudiantes debían describir, en una combinación de lenguaje verbal y matemático, cada uno de los datos consignados en la sección Sintaxis. El profesor abrió este ciclo con la lectura del borrador (la descripción de uno de los datos de la Sintaxis). Y a continuación, mediante las intervenciones 2, 3 y 5 invitó y ayudó a los alumnos a participar en la revisión: pidió diagnosticar (2), evocó un criterio de revisión que previamente habían comentado (precisión conceptual) (3) y pidió una propuesta de solución (5). Esta invitación tuvo impacto en las acciones de los alumnos: A1 diagnosticó el borrador (4), mientras A2, A3 y A4, en tres acciones sucesivas $(6,7$ y 8 ), elaboraron de manera conjunta una propuesta de solución. Finalmente, el profesor confirmó la propuesta de los alumnos (9).
En este ciclo, puede observarse que a diferencia de los ciclos monológicos el profesor actuó de modo reticente (no realizó él mismo las acciones de revisión) y, en cambio, devolvió el derecho y la responsabilidad de la revisión a los estudiantes mediante acciones orientadas a promover su participación: Pide diagnosticar el borrador y Pide propuesta de solución. De igual modo, mediante una acción de Ayuda, en la cual indicó que el texto se alejaba de uno de los criterios de revisión (la precisión), reguló la intervención de los estudiantes. Los alumnos aceptaron la invitación a participar y ejercieron el rol de revisores del borrador.

Desde este rol, se percataron de un aspecto característico de los métodos numéricos: que se trabaja con valores "aproximados" y no exactos, y propusieron una escritura más precisa. La situación de revisión colectiva sirvió a los alumnos, en consecuencia, para tomar conciencia de un rasgo propio de los métodos numéricos a la vez que para ponerse en los zapatos del lector/usuario de la entrada de manual, quien necesita instrucciones inequívocas de parte de quien redacta el manual.

Esta estrategia se repitió en 7 de los 8 ciclos dialógicos de la primera implementación, en 2 de la segunda y en 6 de la tercera.

Tabla 4. Ciclo dialógico, sección Descripción, implementación 1

\begin{tabular}{|c|c|c|c|}
\hline & TRANSCRIPCIÓN & ACCIONES & BORRADOR \\
\hline 1 & $\begin{array}{l}\text { Profesor: Bien, en cuanto a las salidas. En [donde dice] punto } \\
\text { la explicación que está aquí [en el borrador] es Valor de } x \text { tal } \\
\text { que } x=g(x) \text {. }\end{array}$ & Lee el borrador & \\
\hline 2 & P: ¿Qué opinan de esto? & Pide diagnosticar el borrador & \\
\hline 3 & P: Aquí hay una pequeña imprecisión, ¿no es cierto? & $\begin{array}{l}\text { Ayuda (evoca criterio } \\
\text { de revisión: precisión) }\end{array}$ & \\
\hline 4 & $\begin{array}{l}\text { Alumno 1: Sí, porque [el valor de x tal que x] no es igual [a g(x), } \\
\text { sino que es aproximado]. }\end{array}$ & Diagnostica borrador & Valor de y tal loup \\
\hline 5 & P: Porque no es igual. 0 tal vez aquí lo que debiera poner es... & $\begin{array}{l}\text { Confirma } \\
\text { Pide propuesta de solución }\end{array}$ & $x=g(x)$ \\
\hline 6 & A 2: Aproximado. & \multirow{3}{*}{ Propone solución } & \\
\hline 7 & A 3: Aproximación. & & \\
\hline 8 & A 4: Valor aproximado de $x$ tal que $x=g(x)$ & & \\
\hline 9 & P: Sí. "Valor aproximado de x tal que..." & Confirma & \\
\hline
\end{tabular}


Tabla 5. Ciclo dialógico, sección Propósito, implementación 2

\begin{tabular}{|c|c|c|c|}
\hline & TRANSCRIPCIÓN & ACCIONES & BORRADOR \\
\hline 1 & $\begin{array}{l}\text { Profesor: ¿Y en el caso de ustedes? [Se dirige a los miembros de uno } \\
\text { de los grupos y les pregunta qué fue lo que les corrigió el revisor par y } \\
\text { qué opinan de esa corrección]. }\end{array}$ & $\begin{array}{l}\text { Pide leer y/o comen- } \\
\text { tar la revisión del par }\end{array}$ & \multirow{6}{*}{$\begin{array}{l}\text { Propósito: } \\
\text { Eneentrar ceros de } \\
\text { funciones } \\
\text { Encoutrar puntos } \\
\text { fijos }\end{array}$} \\
\hline 2 & $\begin{array}{l}\text { Alumno 1: Igual [que en el borrador del otro grupo, en el nuestro] se ha } \\
\text { corregido el Propósito }\end{array}$ & $\begin{array}{l}\text { Comenta revisión del } \\
\text { par (contenido) }\end{array}$ & \\
\hline 3 & A1: que estaba Encontrar ceros de funciones. & Lee borrador & \\
\hline 4 & $\begin{array}{l}\text { A1: Y poner una tipografía diferente para... [distinguir el lenguaje ver- } \\
\text { bal, del lenguaje de programación y del matemático] }\end{array}$ & $\begin{array}{l}\text { Propone solución } \\
\text { (forma) }\end{array}$ & \\
\hline 5 & P: Eso sí es importante. & Confirma & \\
\hline 6 & $\begin{array}{l}\text { P: Poner tipografía distinta para distinguir entre lenguaje natural, las } \\
\text { palabras comunes y corrientes, y lo que es simbología de Matlab [len- } \\
\text { guaje de programación], y lo que son funciones matemáticas [lenguaje } \\
\text { matemático]. De hecho, debe haber tres tipos de tipografía. }\end{array}$ & Institucionaliza & \\
\hline
\end{tabular}

\section{Incorporar los comentarios} del revisor par realizados previamente

En la segunda implementación, el profesor introdujo una nueva estrategia para promover la participación de los alumnos en la revisión colectiva de borradores: incorporó los comentarios recibidos como parte de la revisión realizada previamente entre los grupos de estudiantes. El ejemplo que analizamos (Tabla 5) forma parte del episodio dedicado a la revisión de la sección Propósito, en la cual debían consignar, en lenguaje verbal, una descripción muy corta del problema que resuelve el método numérico. Como acción de apertura (1), esta vez el profesor optó por pedir a los miembros del grupo autor del borrador que leyeran y comentaran la revisión realizada previamente por el grupo revisor par, que constaba como anotación al margen. A continuación, uno de los miembros leyó el borrador (3) y dos propuestas de solución realizadas por el grupo par, una referida al contenido, que aparece de manera tácita (2), y otra referida a la forma, concretamente, al uso de tipografía diferente, como marca para distinguir los tipos de lenguaje utilizados en la escritura de la entrada de manual (4). El profesor cerró el ciclo con dos acciones: una de confirmación (5) y otra de institucionalización (6). La primera sirvió para avalar la respuesta de los alumnos y la segunda para ampliarla y acercarla al saber erudito.
En este ciclo, el profesor ensayó una nueva estrategia para restituir el derecho a revisar y devolver la responsabilidad a los estudiantes: incorporó a la discusión colectiva, mediante la lectura de los comentarios al margen, la revisión realizada previamente por el grupo par ${ }^{11}$. Se podría objetar el uso de esta estrategia al señalar que el profesor pidió a los alumnos considerar los comentarios recibidos en una actividad de revisión previa, obturando con ello la posibilidad de que pudieran ejercer la revisión in situ. Pero hay que tener en cuenta que la actividad de revisión colectiva no estaba separada de su contexto previo, es decir, de las otras actividades de la secuencia didáctica. Además, los comentarios recibidos del grupo par fueron sometidos a discusión durante esta revisión colectiva, por lo que podrían haber sido rechazados. Al provenir de otros alumnos, y no del profesor, la aceptación de esos comentarios dependía de la persuasión racional que lograran en los autores del texto original, y no exigían su aceptación, como a veces sí suele ocurrir con la voz de autoridad del profesor.

Al incorporar a la revisión colectiva parte del trabajo realizado previamente en la revisión entre pares, el profesor puso en diálogo dos perspectivas divergentes: la del grupo revisor y la del grupo revisado. Al generar esta dialéctica, creó una de las condiciones señaladas por la investigación para aprovechar el potencial epistémico de la escritura.

11 Como se aprecia en la Fig. 1, antes de la revisión colectiva, los estudiantes participaron, en pequeños grupos, de una revisión por pares, consignando comentarios al margen de los borradores. 
La confrontación con el punto de vista del grupo par sirvió como retroacción para cada grupo. Ambos habían atravesado procesos de razonamiento y toma de decisiones en instancias de trabajo previas, tanto el grupo que escribió el borrador como el grupo par que lo revisó. Luego, en la revisión colectiva estas decisiones fueron reconsideradas por el otro, comprometiéndolos en una toma de posición que probablemente catalizó la construcción cognoscitiva.

La interacción entre grupos giró en torno a dos aspectos (uno conceptual y otro formal) relacionados directamente con el objeto de enseñanza: la actividad del usuario de métodos numéricos. Por un lado, se detectó y solucionó un error conceptual que, según señaló el profesor en una entrevista posterior, "podría inducir a error al usuario". Por otro lado, se trató una convención de la entrada de manual relacionada con su carácter multimodal: el uso de diferente tipografía para distinguir cada tipo de lenguaje. Este uso forma parte de los quehaceres del escritor-lector de entradas de manual.
Esta estrategia se repitió en 10 de los 14 ciclos dialógicos de la segunda implementación.

\section{Convocar varios borradores}

Al igual que el anterior, el último ejemplo que analizamos (Tabla 6) también formó parte del episodio dedicado a la revisión de la sección Propósito. Solo que esta vez, en la tercera implementación, el profesor recurrió a una estrategia diferente: convocar varios borradores. Para ilustrarla mejor, hemos incluido, en las filas 1 y 10 con fondo gris (Tabla 6), la última acción del ciclo anterior y la primera del posterior, que también correspondieron al episodio Propósito. En la última acción del ciclo anterior el profesor suspendió la confirmación de la propuesta de solución "hasta oír lo que dicen otros grupos", con lo cual devolvió a los estudiantes la responsabilidad de considerarlas en conjunto (1). Abrió entonces el nuevo ciclo pidiendo a otro grupo leer su borrador, (2). Tras la lectura realizada por Alumno 1, el profesor invitó (4) y luego ayudó (7) a A2 a realizar un diagnóstico del contenido

Tabla 6. Ciclo dialógico, sección Propósito, implementación 3

\begin{tabular}{|c|c|c|c|}
\hline & TRANSCRIPCIÓN & ACCIONES & BORRADOR \\
\hline 1 & $\begin{array}{l}\text { Profesor: pero vamos a dejar esto [la confirmación del ciclo } \\
\text { anterior] en suspenso hasta oír lo que dicen otros grupos. }\end{array}$ & Suspende confirmación & \\
\hline 2 & P: Por ejemplo ustedes, ¿qué dice en Propósito? & Pide leer borrador & \multirow{8}{*}{$\begin{array}{l}\text { Determinar un } x \text { tal } \\
\qquad \text { que } x=g(x)\end{array}$} \\
\hline 3 & Alumno 1: Determinar un $x$ tal que $x=g(x)$. & Lee borrador & \\
\hline 4 & $\begin{array}{l}\text { P: "Determinar un x" .. ¿Qué les parece eso? [Escribe en la } \\
\text { pizarra: Determinar x tal que } x=g(x) \text { ]. Opiniones. ¿Qué dice } \\
\text { usted? ¿Qué le parece? [Se dirige a A2] }\end{array}$ & Pide diagnosticar borrador & \\
\hline 5 & A 2: Yo creo que sí está bien, & Confirma (contenido) & \\
\hline 6 & A 2: pero tal vez es muy... [técnico] & Diagnostica (forma) & \\
\hline 7 & $\begin{array}{l}\text { P: Le parece que está bien, o sea en cuanto a precisión [con- } \\
\text { ceptual], ¿no es cierto? ¿Me está diciendo que está bien en } \\
\text { cuanto a precisión, porque está..., eso es lo que me está dic- } \\
\text { iendo? }\end{array}$ & $\begin{array}{l}\text { Ayuda (evoca criterio de revisión: } \\
\text { precisión) }\end{array}$ & \\
\hline 8 & A 2: Yo creo que sí. Porque esa es la esencia del método. & Valida contenido & \\
\hline 9 & $\begin{array}{l}\text { P: De hecho, esa es la definición de un punto fijo, ¿no es cierto? } \\
0 \text { sea, el punto fijo es un punto } x \text { tal que } x=g(x) \text {. Ese es un } \\
\text { punto fijo de g. Pero está bien, la verdad, yo estoy de acuerdo } \\
\text { con usted. }\end{array}$ & Confirma & \\
\hline 10 & $\begin{array}{l}\text { P: Pero [retoma el diagnóstico del alumno } 2 \text { en el turno 5], la } \\
\text { verdad, hay otra opción [implícitamente sugiere leer otro bor- } \\
\text { rador], ¿no es cierto? }\end{array}$ & Pide leer borrador & \\
\hline
\end{tabular}


del borrador (5, 6 y 8 ) que, finalmente, el profesor confirmó (9). Si bien el contenido de la respuesta era el correcto, este debía estar expresado en leguaje verbal y no en matemático. Para "traducir" la respuesta a lenguaje verbal, el profesor, sin cerrar completamente este ciclo, abrió otro, a partir de un nuevo movimiento de devolución: "hay otra opción" (9), que los alumnos interpretaron como una invitación a leer más borradores.

La estrategia que el profesor empleó en esta ocasión para promover la participación de los estudiantes se basó en el encadenamiento de varios ciclos y en la inclusión, en cada uno de estos ciclos, de una nueva perspectiva (borrador) a ser considerada, contrastada y discutida en el plenario. Esta forma de interacción generó las condiciones para que el conocimiento se elaborara desde la confrontación de varios puntos de vista que, al entrar en tensión, permitieron precisar y sofisticar el pensamiento (Dysthe, 1996).

En este caso, A1, a partir de la lectura del borrador, aportó una solución (elaborada y razonada previamente por los miembros de su grupo) que A2 revisó en dos acciones sucesivas: confirmando el contenido (5) y diagnosticando un problema que, si bien no acabó de formular -"pero tal vez es muy..."-, por el desarrollo posterior de la interacción, podemos inferir hacía referencia al tipo de lenguaje en el cual estaba expresada dicha solución: matemático y no verbal como correspondería (6). Este diagnóstico insinuado por A2 fue retomado por el profesor para mostrar que el problema no se había resuelto del todo y así comenzar un nuevo ciclo que permitiera afinar la respuesta (10).

Vale la pena notar que aquello que se discutió en este ciclo constituye un aspecto central del objeto de enseñanza en la medida que la precisión conceptual con respecto al propósito del método numérico y el manejo de la dimensión multimodal, es decir, del uso pertinente de los diversos lenguajes, constituyen aspectos determinantes de la comprensión del método por parte del lector-usuario de la entrada de manual.

Esta estrategia, que se realizó a partir de poner en revisión varios borradores, incluyó 12 ciclos (dos secuencias de 6 ciclos encadenados) de los 23 ciclos dialógicos de la tercera implementación.

\section{Participación de los alumnos en la construcción de conocimientos}

En los tres ejemplos de ciclos dialógicos que analizamos, se plantearon situaciones de enseñanza a través de un medio, en el cual los alumnos tuvieron ocasión de interactuar tanto con sus textos como con sus pares y con su docente. Para participar, tuvieron que ejercer quehaceres de revisión y movilizar conocimientos de métodos numéricos. En estos casos, la tarea de escritura dejó de ser solo un canal para comunicar al docente lo aprendido, sino que planteó a los alumnos un problema, conceptual y retórico, cuya resolución conllevó formular, justificar y clarificar ideas vinculadas con el objeto de estudio, en función de un propósito de escritura (facilitar el uso de métodos numéricos) y de un destinatario (el usuario). Esto hizo que la actividad realizada en el aula no solo implicara a los alumnos en la producción de un texto apropiado (en lo conceptual y en lo escritural), sino también en refinar su conocimiento del método numérico en cuestión, a la vez que en desarrollarse como revisores de entradas de manual. La devolución y regulación que llevó a cabo el docente a través de diversas estrategias participativas hicieron que la atención dedicada al aspecto productivo de la actividad (dejar escrito un texto adecuado) no relegara su aspecto constructivo (que su realización permita que los alumnos aprendan en el camino). Habría bastado con la corrección del docente y habría sido innecesaria la revisión colectiva participativa si el foco hubiera estado puesto únicamente en lograr un correcto producto escrito.

El hecho de que las intervenciones de los estudiantes fueran inacabadas, parcialmente erróneas o contradictorias, lejos de ser un obstáculo, ayudó a crear las condiciones que posibilitaron la construcción de conocimientos mediante la formulación de ideas, su discusión y argumentación. El significado no surgió del pensamiento de un solo individuo, sino del diálogo que se estableció entre un colectivo con conocimientos diversos, pero animados por un mismo objetivo. En los ciclos dialógicos, el aporte del docente se replegó para dar cabida al aporte de los estudiantes. La reticencia, devolución y regulación del docente permitieron la confrontación de interpretaciones, dando pie a la construcción de nuevos significados, ya sea porque los estudiantes incorporaron una idea ajena, porque reafirmaron la propia a partir de contrastarla con otra, porque desarrollaron una novedosa a partir de esta dialéctica 
o porque debieron buscar una justificación que validara su postura. Es improbable que esta actividad cognoscitiva de los estudiantes tenga oportunidad de desplegarse cuando el profesor es el único responsable de formular y validar saberes.

Cabe notar que la decisión de atender al potencial epistémico de la escritura es lo que determinó prever la participación de los alumnos en la revisión colectiva, incluso a costa de sacrificar un avance rápido de los temas trabajados. De este modo, el tiempo didáctico se ralentizó, al punto de impedir que el episodio Ejemplos pudiera ser tratado en la tercera implementación. Sin embargo, a los fines de entender que este avance lento en la progresión de los contenidos tratados en clase no significó un enlentecimiento en los conocimientos aprendidos, cabe preguntarse por la inversa (¿si se hubiera acelerado el abordaje de los temas mediante la modalidad expositiva, se habría acelerado su aprendizaje?). Para responder el interrogante conviene recordar que el tiempo del aprendizaje no se corresponde con el tiempo de la enseñanza (Lerner, 2017).

\subsubsection{Exposición del profesor y participación de los alumnos}

A pesar del incremento de los ciclos dialógicos, es dable notar que el docente alternó entre una estrategia expositiva y tres participativas. La primera fue útil para compartir información y modelizar un quehacer de revisión y, de este modo, para hacer avanzar a mayor ritmo los contenidos trabajados. Las segundas sirvieron para devolver a los estudiantes su derecho y responsabilidad en la revisión del texto al tiempo que facilitaron que la escritura funcionara como herramienta de aprendizaje.

Además, cuando los estudiantes participaron del diálogo plural el profesor recibió información sobre lo que iban comprendiendo del tema. Como contrapartida, el tiempo didáctico se volvió escaso al dar cabida a esta participación y, por ello, en la tercera implementación no pudieron abordarse todos los contenidos previstos. Mientras en los ciclos monológicos el profesor tuvo un mayor control de los contenidos y del tiempo didáctico, en los dialógicos cedió parte de este control a los alumnos y probablemente contribuyó a su comprensión y aprendizaje.

Finalmente, conviene notar que los ciclos dialógicos, si bien muestran menor protagonismo del docente en tanto expositor, se lo devuelven en tanto requieren de su acción didáctica para abrir el juego a la participación de los alumnos. Es otro tipo de protagonismo el del profesor cuando sabe que él es responsable de gestionar la interacción entre docente-alumnos-borradores.

\section{Conclusiones}

En este artículo hemos caracterizado, a partir de transcripciones de clase, las estrategias que ensayó un profesor de Métodos numéricos para favorecer la participación de sus alumnos en el diálogo sobre lo escrito al implementar, a lo largo de tres semestres, una secuencia didáctica codiseñada entre este y los investigadores. Antes de esta intervención, los alumnos escribían individualmente fuera de clase y el profesor era quien corregía sus textos. Con el propósito de que la escritura sirviera epistémicamente, es decir, como instrumento para ayudar a acrecentar la comprensión sobre las nociones estudiadas en esta asignatura de Ingeniería, se decidió incluir en clase una tarea de revisión entre grupos de pares y otra de revisión colectiva de borradores de una entrada de manual de métodos numéricos. Las condiciones para aprovechar la función epistémica de la escritura que se intentaron crear (intercambios plurales con controversia académica, que promovieran la reconsideración de ideas) estuvieron alineadas con las condiciones didácticas necesarias para que los alumnos se involucrasen en la construcción de conocimientos y no fueran meros receptores del saber expuesto por el profesor.

El análisis realizado en las páginas previas sobre la revisión colectiva muestra una coexistencia entre estrategias expositivas y participativas llevadas a cabo por el docente, con un aumento progresivo de las segundas a lo largo de las tres implementaciones de la secuencia. Esta tendencia longitudinal, junto con la experimentación de diversas estrategias participativas (Invitar a revisar, Incorporar los comentarios del revisor par realizados previamente, Convocar varios borradores) por parte del profesor, indica una paulatina transformación en su actividad, probablemente favorecida por las entrevistas de autoconfrontación realizadas con el primer autor.

Las estrategias que denominamos participativas exhiben que el docente compartió con sus alumnos el rol de revisor y les restituyó su parte de la responsabilidad, a la vez que su derecho, en la construcción de significados. Hemos mostrado que las tres estrategias participativas identificadas 
en este estudio contienen dos movimientos del profesor: por un lado, su reticencia y por otro lado, la devolución y la regulación. Mediante el movimiento de reticencia, se inhibió de realizar la revisión él mismo. Complementariamente, el movimiento de devolución y regulación habilitó a los alumnos a ejercer los quehaceres de revisión (diagnosticar los problemas del texto y proponer mejoras), a la vez que les permitió formular y justificar sus conocimientos sobre métodos numéricos, en proceso de construcción. Concomitantemente, posibilitó que el profesor tomara conciencia de lo que iban comprendiendo sus estudiantes.

Si en los ciclos monológicos (característicos de la estrategia expositiva) la actividad del profesor se centró en el ejercicio de acciones de revisión, que cumplieran una función informativa o demostrativa para los alumnos, en los ciclos dialógicos (propios de las estrategias participativas) la actividad del profesor se concentró en realizar acciones de carácter apelativo, en el sentido de que buscaban provocar la realización de acciones de revisión por parte de los alumnos. En los ciclos dialógicos, las acciones del profesor dejaron de tener importancia por lo que hicieron sobre los textos y pasaron a tener importancia por lo que permitieron a los estudiantes hacer sobre ellos (mejorarlos) y a partir de ello (mejorar como escritores de entradas de manual).

Este cambio implicó a su vez una modificación del contrato didáctico y de los roles que el profesor y los estudiantes esperaban del otro. Mientras en los ciclos monológicos el profesor esperaba que los alumnos escuchasen y observasen cómo se revisa, en los dialógicos esperaba que los alumnos revisasen. $Y$ a la inversa, mientras en los monológicos los alumnos esperaban que el profesor revisara, en los dialógicos esperaban que los ayudara a revisar.

En definitiva, nuestro estudio aporta a profundizar lo que se entiende por "aprovechar la función epistémica de la escritura" en la educación. En el caso explorado, consistió en crear situaciones de trabajo en clase que auspiciaran el diálogo plural sobre lo escrito, en línea con ciertas condiciones didácticas que Brousseau (2007), Dysthe et. al. (2013), Lerner (2001, 2017) y Sensevy (2007) sugieren sostener para que los diversos alumnos participen en la construcción del conocimiento. Ha de notarse que las situaciones creadas para que los estudiantes protagonicen su aprendizaje en clase no restan protagonismo al profesor sino, por el contrario, requieren de su acción para configurar un medio y un contrato didáctico inhabituales, con tareas, roles y expectativas mutuas diferentes a la usual clase expositiva, mediante movimientos de reticencia, devolución y regulación. .

\section{Referencias bibliográficas}

Artigue, M. (1995). "Ingeniería didáctica" en Artigue, M., Douady, R., Moreno, L., Gómez, P. (Ed.) Ingeniería didáctica en educación matemática. Bogotá: Una empresa docente \& Grupo Editorial Iberoamérica, pp. 33-59.

Bajtin, M. (2012). Problemas de la poética de Dostoievski, $3^{\mathrm{a}}$ ed. México: Fondo de Cultura Económica.

Bazerman, C., Little, J., Bethel, L., Chavkin, T., Fouquette, D. y Garufis, J. (2005). Reference Guide to Writing Across the Curriculum. West Lafayette, Indiana: Parlor Press and The WAC Clearinghouse.

Brousseau, G. (2007). Iniciación al estudio de la teoría de las situaciones didácticas, 1a ed. Buenos Aires: Libros del Zorzal.

Carlino, P. (2004). La distancia que separa la evaluación escrita frecuente de la deseable. Acción Pedagógica, 13 (1), 8-17. https://mww.aacademica.org/paula. carlino/123.pdf

Carlino, P.; Iglesia, P. y Laxalt, I. (2013). Concepciones y prácticas declaradas de profesores terciarios en torno al leer y escribir en las asignaturas. Revista de Docencia Universitaria, 11(1), 105-135. https:// www.aacademica. org/paula.carlino/215. pdf/

Carter, M., Ferzli, M. y Wiebe, E. N. (2007). Writing to learn by learning to write in the disciplines. Journal of Business and Technical Communication, 21(3), 278-302.

Dysthe, O. (1996). The Multivoiced Classroom: Interactions of Writing and Classroom Discourse. Written Communication, 13(3), 385-425.

Dysthe, O., Bernhardt, N, y Esbjørn, L. (2013). Enseñanza basada en el $n$. Skoletjenesten: Copenhague.

Fernández, G., y Clot, Y. (2010) Entrevistas en autoconfrontación: un método en clínica de la actividad. Informática na Educação: teoria \& prática. 13(1), 11-16.

Klein, P. D. (1999). Reopening inquiry into cognitive processes in writing-to-learn. Educational Psychology Review, 11(3), 203-270. 
Langer, J. A. y Applebee, A. N. (2007). How Writing Shapes Thinking: A Study of Teaching and Learning. WACClearinghouse LandmarkPublicationsinWriting Studies. Recuperado en: http://wac.colostate.edu/ books/langer_applebee/ Originalmente publicado impreso en 1987 por el National Council of Teachers of English, Urbana, Illinois.

Lerner, D. (2001). Leer y escribir en la escuela: Io real, lo posible y lo necesario. México: Fondo de Cultura Económica.

Lerner, D. (2017). Diversidad (es) e inclusión educativa. Unamirada desdelas Didácticas Específicas. Video de la conferencia en ocasión del Primer Congreso de Educación Diferencial con foco en inclusión escolar, Universidad Alberto Hurtado, abril 2017. Recuperado en enero de 2019 de https://www. youtube. $. c 0 m /$ watch?V=Wn4JR5P57ZA

Maxwell, J. A. (1996). Qualitative Research Design. An interactive Approach. Thousand Oaks: SAG Publications.

Ochsner, R. y Fowler, J. (2004). Playing Devil's Advocate: Evaluating the Literature of the WAC/ WID Movement. Review of Educational Research, 74 (2), 117-140.

Pastré, P. (2007). Quelques réflexions sur l'organisation de l'activité enseignante [Traducción de Elisabeth Muñoz de Corrales]. Recherche et formation [En ligne], 56. Disponible en : https://journals. openedition. org/rechercheformation/907

Pastré, P. (2008). Apprentissage et activité. En Y. Lenoir y P. Pastré (Eds.), Didactique professionnelle et didactiques disciplinaires en débat: un enjeu pour la professionnalisation des enseignants. [Traducción de Elisabeth Muñoz de Corrales]. Toulouse: Octarès Éditions, 53-79.

Pastré, P. (2011). "Situación de aprendizaje y conceptualización» en Recherches en Education, $N^{\circ} 12$ - « Revisiter la notion de situation: approches plurielles » p. 12-25. Coordonné par Yves LENOIR et Frédéric TUPIN. Traducido del francés por Elisabeth Muñoz de Corrales (UNIPE).

Sánchez, E., García, R. y Rosales, J. (2010). La lectura en el aula. Qué se hace, qué se debe hacer y qué se puede hacer. Barcelona: GRAÓ.

Sensevy, G. (2007). Categorías para describir y comprender la acción didáctica. En Sensevy, G y Mercier, A. (2007). Agir ensemble: l'action didactique conjointe du professeur et des élèves.
Rennes: PUR. 5-34. Traducción de Juan Duque y revisión de René Rickenmann. Programa de colaboración de la Universidad de Antioquia y de la Universidad de Ginebra, y sus grupos de investigación GECEM-SED 2007-2009.

Sensevy, G., Forest, D., Quilio, S. y Morales, G. (2013). Cooperative engineering as a specific design-based research. ZDM, The International Journal on Mathematics Education, 45(7), pp. 1031-1043.

Wells, G. (1990). Creating the Conditions to Encourage Literate Thinking. Educational Leadership, 47(6), 13-17.

Wells, G. (2004). Dialogic Inquiry. Toward a Sociocultural Practice and Theory of Education. Cambridge University Press. 